\title{
BIPOLAR-VALUED FUZZY IDEAL EXTENSIONS OF BCK-ALGEBRAS
}

\author{
WON KYUn JEONG*
}

\begin{abstract}
The notions of the extensions of bipolar fuzzy ideals, bipolar fuzzy prime ideals and bipolar fuzzy commutative ideals in BCK-algebras are introduced, and several properties are investigated.
\end{abstract}

\section{Introduction}

In 1966, Y. Imai and K. Iséki [1] introduced a notion called a BCKalgebra, and since then many researchers have investigated various properties of this algebra. Such algebra generalized the notion of sets with the set subtraction as the only nonnullary operation. The concept of fuzzy sets was introduced by L. A. Zadeh [10]. Since then fuzzy sets have been applied to other algebraic structures such as semigroups, groups and rings, etc. O. G. Xi [8] applied the concept of fuzzy set to BCKalgebras, and he investigated some results.

K. M. Lee [6] introduced the notion of bipolar-valued fuzzy sets which is an extension of fuzzy sets. K. J. Lee [5] introduced the notion of bipolar fuzzy subalgebras/ideals of a BCK-algebra, and investigated several properties.

In this paper, we introduce the notion of extensions of bipolar fuzzy ideals in BCK-algebras, and investigate some related properties of it. We also introduce the notion of bipolar fuzzy commutative ideals in BCK-algebras, and provide conditions for a bipolar fuzzy ideal to be a bipolar fuzzy commutative ideal.

Received November 11, 2012; Accepted January 11, 2013.

2010 Mathematics Subject Classification: Primary 06F35, 03G25.

Key words and phrases: bipolar fuzzy set, bipolar fuzzy ideal, positive implicative BCK-algebra. 


\section{Preliminaries}

We begin with the following well-known definitions and results which are necessary for completeness.

An algebra $(X ; *, 0)$ of type $(2,0)$ is called a $B C K$-algebra if for all $x, y, z \in X$ the following conditions hold:

(a) $((x * y) *(x * z)) *(z * y)=0$,

(b) $(x *(x * y)) * y)=0$,

(c) $x * x=0$,

(d) $0 * x=0$,

(e) $x * y=0$ and $y * x=0$ imply $x=y$.

We can define a partial ordering " $\leq$ " on $X$ by $x \leq y$ if and only if $x * y=0$. Any BCK-algebra $X$ satisfies the following axioms:

(1) $(\forall x \in X)(x * 0=x)$,

(2) $(\forall x, y, z \in X)(x \leq y \Rightarrow x * z \leq y * z, z * y \leq z * x)$,

(3) $(\forall x, y, z \in X)((x * y) * z=(x * z) * y)$,

(4) $(\forall x, y, z \in X)((x * z) *(y * z) \leq x * y)$.

A nonempty subset $S$ of a BCK-algebra $X$ is called a subalgebra of $X$ if $x * y \in S$ whenever $x, y \in S$. A subset $A$ of a BCK-algebra $X$ is called an ideal of $X$ if it satisfies the following conditions:

(I1) $0 \in A$,

(I2) $(\forall x, y \in X)(x * y \in A, y \in A \Rightarrow x \in A)$.

Now we review some fuzzy logic concepts. A fuzzy set in a set $S$ is a function $\mu: S \rightarrow[0,1]$. For any fuzzy sets $\mu$ and $\nu$ in $S$, we define

$$
\mu \subseteq \nu \Leftrightarrow \mu(x) \leq \nu(x) \text { for all } x \in S .
$$

A fuzzy set $\mu$ in a BCK-algebra $X$ is called a fuzzy subalgebra of $X$ if $\mu(x * y) \geq \min \{\mu(x), \mu(y)\}$ for all $x, y \in X$. A fuzzy set $\mu$ in a BCK-algebra $X$ is called a fuzzy ideal of $X$ if it satisfies the following conditions:

$(F I 1)(\forall x \in X)(\mu(0) \geq \mu(x))$,

(FI2) $(\forall x, y \in X)(\mu(x) \geq \min \{\mu(x * y), \mu(y)\})$.

K. M. Lee [6] introduced the notion of bipolar-valued fuzzy sets which is an extension of fuzzy sets. Let $X$ be the universe of discourse. A bipolar-valued fuzzy set $\Psi$ in $X$ is an object having the form

$$
\Psi=\left\{\left(x, \mu_{\Psi}^{P}(x), \mu_{\Psi}^{N}(x)\right) \mid x \in X\right\},
$$

where $\mu_{\Psi}^{P}: X \rightarrow[0,1]$ and $\mu_{\Psi}^{N}: X \rightarrow[-1,0]$ are mappings. The positive membership degree $\mu_{\Psi}^{P}(x)$ denotes the satisfaction degree of an 
element $x$ to the property corresponding to a bipolar-valued fuzzy set $\Psi=\left\{\left(x, \mu_{\Psi}^{P}(x), \mu_{\Psi}^{N}(x)\right) \mid x \in X\right\}$, and the negative membership degree $\mu_{\Psi}^{N}(x)$ denotes the satisfaction degree of $x$ to some implicit counterproperty of $\Psi=\left\{\left(x, \mu_{\Psi}^{P}(x), \mu_{\Psi}^{N}(x)\right) \mid x \in X\right\}$. We shall use the symbol $\Psi=\left(X ; \mu_{\Psi}^{P}, \mu_{\Psi}^{N}\right)$ for the bipolar-valued fuzzy set $\Psi=\left\{\left(x, \mu_{\Psi}^{P}(x), \mu_{\Psi}^{N}(x)\right)\right.$ $\mid x \in X\}$, and use the notion of biploar fuzzy sets instead of the notion of bipolar-valued fuzzy sets. For any bipolar fuzzy sets $\Psi_{1}=\left(X ; \mu_{\Psi_{1}}^{P}, \mu_{\Psi_{1}}^{N}\right)$ and $\Psi_{2}=\left(X ; \mu_{\Psi_{2}}^{P}, \mu_{\Psi_{2}}^{N}\right)$ in $X$, we define

$$
\Psi_{1} \subseteq \Psi_{2} \Leftrightarrow \mu_{\Psi_{1}}^{P}(x) \leq \mu_{\Psi_{2}}^{P}(x) \text { and } \mu_{\Psi_{1}}^{N}(x) \geq \mu_{\Psi_{2}}^{N}(x) \text { for all } x \in X .
$$

Definition 2.1. ([5]) A bipolar fuzzy set $\Psi=\left(X ; \mu_{\Psi}^{P}, \mu_{\Psi}^{N}\right)$ in a BCK-algebra $X$ is called a bipolar fuzzy subalgebra of $X$ if it satisfies:

(BS1) $\mu_{\Psi}^{P}(x * y) \geq \min \left\{\mu_{\Psi}^{P}(x), \mu_{\Psi}^{P}(y)\right\}$,

$(B S 2) \mu_{\Psi}^{N}(x * y) \leq \max \left\{\mu_{\Psi}^{N}(x), \mu_{\Psi}^{N}(y)\right\}$,

for all $x, y \in X$.

Proposition 2.2. ([5]) Let $\Psi=\left(X ; \mu_{\Psi}^{P}, \mu_{\Psi}^{N}\right)$ be a bipolar fuzzy subalgebra of a $B C K$-algebra $X$. Then $\mu_{\Psi}^{P}(0) \geq \mu_{\Psi}^{P}(x)$ and $\mu_{\Psi}^{N}(0) \leq$ $\mu_{\Psi}^{N}(x)$, for all $x \in X$.

Definition 2.3. ([5]) A bipolar fuzzy set $\Psi=\left(X ; \mu_{\Psi}^{P}, \mu_{\Psi}^{N}\right)$ in a BCK-algebra $X$ is called a bipolar fuzzy ideal of $X$ if it satisfies:

(BI1) $\mu_{\Psi}^{P}(0) \geq \mu_{\Psi}^{P}(x)$ and $\mu_{\Psi}^{N}(0) \leq \mu_{\Psi}^{N}(x)$,

(BI2) $\mu_{\Psi}^{P}(x) \geq \min \left\{\mu_{\Psi}^{P}(x * y), \mu_{\Psi}^{P}(y)\right\}$,

(BI3) $\mu_{\Psi}^{N}(x) \leq \max \left\{\mu_{\Psi}^{N}(x * y), \mu_{\Psi}^{N}(y)\right\}$,

for all $x, y \in X$.

Theorem 2.4. ([5]) Every bipolar fuzzy ideal of a BCK-algebra $X$ is a bipolar fuzzy subalgebra of $X$.

Lemma 2.5. ([5]) If $\Psi=\left(X ; \mu_{\Psi}^{P}, \mu_{\Psi}^{N}\right)$ is a bipolar fuzzy ideal of a $B C K$-algebra $X$ and if $x \leq y$ in $X$, then $\mu_{\Psi}^{P}(x) \geq \mu_{\Psi}^{P}(y)$ and $\mu_{\Psi}^{N}(x) \leq$ $\mu_{\Psi}^{N}(y)$.

\section{Bipolar fuzzy ideal extensions}

In what follows, $X$ would mean a BCK-algebra unless otherwise specipied. 
Definition 3.1. Let $\Psi=\left(X ; \mu_{\Psi}^{P}, \mu_{\Psi}^{N}\right)$ be a bipolar fuzzy set of $X$, and let $a, b \in X$. The extension $\langle\Psi,(a, b)\rangle$ of $\Psi$ by $(a, b)$ is an object having the form

$$
\langle\Psi,(a, b)\rangle=\left\{\left(x,\langle\mu, a\rangle_{\Psi}^{P}(x),\langle\mu, b\rangle_{\Psi}^{N}(x)\right) \mid x \in X\right\},
$$

where $\langle\mu, a\rangle_{\Psi}^{P}: X \rightarrow[0,1]$ and $\langle\mu, b\rangle_{\Psi}^{N}: X \rightarrow[-1,0]$ are mappings defined by

$$
\langle\mu, a\rangle_{\Psi}^{P}(x)=\mu_{\Psi}^{P}(x * a) \text { and }\langle\mu, b\rangle_{\Psi}^{N}(x)=\mu_{\Psi}^{N}(x * b),
$$

for all $x \in X$, respectively. If $a=b$, we write $\langle\Psi, a\rangle$ in place of $\langle\Psi,(a, a)\rangle$.

Note that $\langle\Psi,(a, b)\rangle$ is a bipolar fuzzy set of $X$.

Proposition 3.2. Let $\Psi=\left(X ; \mu_{\Psi}^{P}, \mu_{\Psi}^{N}\right)$ be a bipolar fuzzy ideal of $X$. If $a \leq b$ and $c \leq d$ in $X$, then $\langle\Psi,(a, c)\rangle \subseteq\langle\Psi,(b, d)\rangle$.

Proof. Let $a, b, c, d \in X$. If $a \leq b$ and $c \leq d$, then we have $x * a \geq x * b$ and $x * c \geq x * d$, for all $x \in X$. Since $\Psi=\left(X ; \mu_{\Psi}^{P}, \mu_{\Psi}^{N}\right)$ is a bipolar fuzzy ideal of $X$, it follows from Lemma 2.5 that $\langle\mu, a\rangle_{\Psi}^{P}(x)=\mu_{\Psi}^{P}(x * a) \leq$ $\mu_{\Psi}^{P}(x * b)=\langle\mu, b\rangle_{\Psi}^{P}(x)$ and $\langle\mu, c\rangle_{\Psi}^{N}(x)=\mu_{\Psi}^{N}(x * c) \geq \mu_{\Psi}^{N}(x * d)=$ $\langle\mu, d\rangle_{\Psi}^{N}(x)$, for all $x \in X$. Thus, we have $\langle\Psi,(a, c)\rangle \subseteq\langle\Psi,(b, d)\rangle$.

Definition 3.3. ([2]) A BCK-algebra $X$ is said to be positive implicative if it satisfies:

$$
(x * y) * z=(x * z) *(y * z),
$$

for all $x, y, z \in X$.

TheOREM 3.4. Let $X$ be a positive implicative BCK-algebra and let $a, b \in X$. If $\Psi=\left(X ; \mu_{\Psi}^{P}, \mu_{\Psi}^{N}\right)$ is a bipolar fuzzy subalgebra of $X$, then the extension $\langle\Psi,(a, b)\rangle=\left(X ;\langle\mu, a\rangle_{\Psi}^{P},\langle\mu, b\rangle_{\Psi}^{N}\right)$ of $\Psi$ by $(a, b)$ is a bipolar fuzzy subalgebra of $X$.

Proof. Let $\Psi=\left(X ; \mu_{\Psi}^{P}, \mu_{\Psi}^{N}\right)$ be a bipolar fuzzy subalgebra of a positive implicative BCK-algebra $X$ and let $a, b \in X$. Then

$$
\begin{aligned}
\langle\mu, a\rangle_{\Psi}^{P}(x * y) & =\mu_{\Psi}^{P}((x * y) * a) \\
& =\mu_{\Psi}^{P}((x * a) *(y * a)) \\
& \geq \min \left\{\mu_{\Psi}^{P}(x * a), \mu_{\Psi}^{P}(y * a)\right\} \\
& =\min \left\{\langle\mu, a\rangle_{\Psi}^{P}(x),\langle\mu, a\rangle_{\Psi}^{P}(y)\right\}
\end{aligned}
$$


and

$$
\begin{aligned}
\langle\mu, b\rangle_{\Psi}^{N}(x * y) & =\mu_{\Psi}^{N}((x * y) * b) \\
& =\mu_{\Psi}^{N}((x * b) *(y * b)) \\
& \leq \max \left\{\mu_{\Psi}^{N}(x * b), \mu_{\Psi}^{N}(y * b)\right\} \\
& =\max \left\{\langle\mu, b\rangle_{\Psi}^{N}(x),\langle\mu, b\rangle_{\Psi}^{N}(y)\right\}
\end{aligned}
$$

for all $x, y \in X$. Thus, $\langle\Psi,(a, b)\rangle=\left(X ;\langle\mu, a\rangle_{\Psi}^{P},\langle\mu, b\rangle_{\Psi}^{N}\right)$ is a bipolar fuzzy subalgebra of $X$. This completes the proof.

TheOREM 3.5. Let $X$ be a positive implicative BCK-algebra and let $\Psi=\left(X ; \mu_{\Psi}^{P}, \mu_{\Psi}^{N}\right)$ be a bipolar fuzzy ideal of $X$. Then the extension $\langle\Psi,(a, b)\rangle=\left(X ;\langle\mu, a\rangle_{\Psi}^{P},\langle\mu, b\rangle_{\Psi}^{N}\right)$ of $\Psi$ by $(a, b)$ is a bipolar fuzzy ideal of $X$.

Proof. Let $\Psi=\left(X ; \mu_{\Psi}^{P}, \mu_{\Psi}^{N}\right)$ be a bipolar fuzzy ideal of a positive implicative BCK-algebra $X$ and let $a, b \in X$. Since $\mu_{\Psi}^{P}(0) \geq \mu_{\Psi}^{P}(x)$ for all $x \in X$, we have

$$
\begin{aligned}
\langle\mu, a\rangle_{\Psi}^{P}(0) & =\mu_{\Psi}^{P}(0 * a) \\
& =\mu_{\Psi}^{P}(0) \\
& \geq \mu_{\Psi}^{P}(x * a) \\
& =\langle\mu, a\rangle_{\Psi}^{P}(x)
\end{aligned}
$$

and

$$
\begin{aligned}
\langle\mu, b\rangle_{\Psi}^{N}(0) & \left.=\mu_{\Psi}^{N}(0 * b)\right) \\
& =\mu_{\Psi}^{N}(0) \\
& \leq \mu_{\Psi}^{N}(x * b) \\
& =\langle\mu, b\rangle_{\Psi}^{N}(x),
\end{aligned}
$$

for all $x \in X$. If $x, y \in X$, then

$$
\begin{aligned}
\langle\mu, a\rangle_{\Psi}^{P}(x) & =\mu_{\Psi}^{P}(x * a) \\
& \geq \min \left\{\mu_{\Psi}^{P}((x * a) *(y * a)), \mu_{\Psi}^{P}(y * a)\right\} \\
& =\min \left\{\mu_{\Psi}^{P}((x * y) * a), \mu_{\Psi}^{P}(y * a)\right\} \\
& =\min \left\{\langle\mu, a\rangle_{\Psi}^{P}(x * y),\langle\mu, a\rangle_{\Psi}^{P}(y)\right\},
\end{aligned}
$$


and

$$
\begin{aligned}
\langle\mu, b\rangle_{\Psi}^{N}(x) & =\mu_{\Psi}^{N}(x * b) \\
& \leq \max \left\{\mu_{\Psi}^{N}((x * b) *(y * b)), \mu_{\Psi}^{N}(y * b)\right\} \\
& =\max \left\{\mu_{\Psi}^{N}((x * y) * b), \mu_{\Psi}^{N}(y * b)\right\} \\
& =\max \left\{\langle\mu, b\rangle_{\Psi}^{N}(x * y),\langle\mu, b\rangle_{\Psi}^{N}(y)\right\} .
\end{aligned}
$$

This shows that $\langle\Psi,(a, b)\rangle=\left(X ;\langle\mu, a\rangle_{\Psi}^{P},\langle\mu, b\rangle_{\Psi}^{N}\right)$ is a bipolar fuzzy ideal of $X$. This completes the proof.

In the above Theorem, "positive implicative" is essential as the following example shows.

ExAmple 3.6. Let $X=\{0,1,2,3,4\}$ in which $*$ is defined by:

\begin{tabular}{l|lllll}
$*$ & 0 & 1 & 2 & 3 & 4 \\
\hline 0 & 0 & 0 & 0 & 0 & 0 \\
1 & 1 & 0 & 1 & 0 & 1 \\
2 & 2 & 2 & 0 & 0 & 0 \\
3 & 3 & 3 & 3 & 0 & 3 \\
4 & 4 & 4 & 2 & 2 & 0
\end{tabular}

Then $(X ; *, 0)$ is a BCK-algebra $([4])$, but not positive implicative, since $(4 * 2) * 3=0$ and $(4 * 3) *(2 * 3)=2$. Let $\Psi=\left(X ; \mu_{\Psi}^{P}, \mu_{\Psi}^{N}\right)$ be the bipolar fuzzy set in $X$ defined by

$$
\mu_{\Psi}^{P}=\left(\begin{array}{ccccc}
0 & 1 & 2 & 3 & 4 \\
1 & 0.5 & 0.2 & 0.1 & 0.2
\end{array}\right)
$$

and

$$
\mu_{\Psi}^{N}=\left(\begin{array}{ccccc}
0 & 1 & 2 & 3 & 4 \\
-0.8 & -0.5 & -0.3 & -0.1 & -0.3
\end{array}\right) .
$$

Then, by routine calculations, it is a bipolar fuzzy ideal of $X$. The extension $\langle\Psi, 3\rangle=\left(X ;\langle\mu, 3\rangle_{\Psi}^{P},\langle\mu, 3\rangle_{\Psi}^{N}\right)$ of $\Psi$ by 3 , however, is not a bipolar fuzzy ideal of $X$, since $\langle\mu, 3\rangle_{\Psi}^{P}(4)=0.2 \nsupseteq 1=\min \left\{\langle\mu, 3\rangle_{\Psi}^{P}(4 *\right.$ 2), $\left.\langle\mu, 3\rangle_{\Psi}^{P}(2)\right\}$.

Definition 3.7. ([2]) A BCK-algebra $X$ is said to be implicative if for all $x, y \in X$,

$$
x=x *(y * x) .
$$

Definition 3.8. ([2]) A BCK-algebra $X$ is said to be commutative if it satisfies

$$
x *(x * y)=y *(y * x),
$$


for all $x, y \in X$.

Note that every subalgebra of a commutative BCK-algebra is also commutative.

Theorem 3.9. ([2]) A BCK-algebra is implicative if and only if it is both commutative and positive implicative.

Definition 3.10. A bipolar fuzzy set $\Psi=\left(X ; \mu_{\Psi}^{P}, \mu_{\Psi}^{N}\right)$ is said to be non-constant if both $\mu_{\Psi}^{P}$ and $\mu_{\Psi}^{N}$ are non-constant mappings.

Definition 3.11. A non-constant bipolar fuzzy ideal $\Psi=\left(X ; \mu_{\Psi}^{P}, \mu_{\Psi}^{N}\right)$ of a commutative BCK-algebra $X$ is said to be prime if for all $x, y \in X$,

$(P 1) \mu_{\Psi}^{P}(x \wedge y) \leq \max \left\{\mu_{\Psi}^{P}(x), \mu_{\Psi}^{P}(y)\right\}$,

$(P 2) \mu_{\Psi}^{N}(x \wedge y) \geq \min \left\{\mu_{\Psi}^{N}(x), \mu_{\Psi}^{N}(y)\right\}$,

where $x \wedge y=y *(y * x)$.

Since $x \wedge y \leq x, y$ and since $\mu_{\Psi}^{P}$ is order reversing and $\mu_{\Psi}^{N}$ is order preserving, it follows that $\mu_{\Psi}^{P}(x) \leq \mu_{\Psi}^{P}(x \wedge y), \mu_{\Psi}^{P}(y) \leq \mu_{\Psi}^{P}(x \wedge y), \mu_{\Psi}^{N}(x) \geq$ $\mu_{\Psi}^{N}(x \wedge y)$ and $\mu_{\Psi}^{N}(y) \geq \mu_{\Psi}^{N}(x \wedge y)$. Hence a non-constant bipolar fuzzy ideal $\Psi=\left\langle X, \mu_{\Psi}^{P}, \mu_{\Psi}^{N}\right\rangle$ is bipolar fuzzy prime if and only if $\mu_{\Psi}^{P}(x \wedge$ $y)=\max \left\{\mu_{\Psi}^{P}(x), \mu_{\Psi}^{P}(y)\right\}$ and $\mu_{\Psi}^{N}(x \wedge y)=\min \left\{\mu_{\Psi}^{N}(x), \mu_{\Psi}^{N}(y)\right\}$, for all $x, y \in X$.

ExAmple 3.12 . Let $X=\{0, a, b, c, d\}$ in which $*$ is defined by :

\begin{tabular}{c|ccccc}
$*$ & 0 & $a$ & $b$ & $c$ & $d$ \\
\hline 0 & 0 & 0 & 0 & 0 & 0 \\
$\mathrm{a}$ & $a$ & 0 & $a$ & $a$ & 0 \\
$\mathrm{~b}$ & $b$ & $b$ & 0 & 0 & 0 \\
$\mathrm{c}$ & $c$ & $c$ & $b$ & 0 & $b$ \\
$\mathrm{~d}$ & $d$ & $d$ & $a$ & $a$ & 0
\end{tabular}

Then $(X ; *, 0)$ is a commutative BCK-algebra. Define mappings $\mu_{\Psi}^{P}$ : $X \rightarrow[0,1]$ and $\mu_{\Psi}^{N}: X \rightarrow[-1,0]$ by

$$
\mu_{\Psi}^{P}=\left(\begin{array}{ccccc}
0 & 1 & 2 & 3 & 4 \\
1 & 0.8 & 0.5 & 0.5 & 0.5
\end{array}\right)
$$

and

$$
\mu_{\Psi}^{N}=\left(\begin{array}{ccccc}
0 & 1 & 2 & 3 & 4 \\
-1 & -0.8 & -0.4 & -0.4 & -0.4
\end{array}\right) .
$$

By routine calculations we know that $\Psi=\left(X ; \mu_{\Psi}^{P}, \mu_{\Psi}^{N}\right)$ is a bipolar fuzzy ideal of $X$, but not a bipolar fuzzy prime ideal of $X$ because

$$
\mu_{\Psi}^{P}(a \wedge b)=1 \not \leq 0.8=\max \left\{\mu_{\Psi}^{P}(a), \mu_{\Psi}^{P}(b)\right\} .
$$


Theorem 3.13. Let $\Psi=\left(X ; \mu_{\Psi}^{P}, \mu_{\Psi}^{N}\right)$ be a bipolar fuzzy prime ideal of an implicative BCK-algebra $X$ and let $a, b \in X$. If the extension $\langle\Psi,(a, b)\rangle=\left(X ;\langle\mu, a\rangle_{\Psi}^{P},\langle\mu, b\rangle_{\Psi}^{N}\right)$ of $\Psi$ by $(a, b)$ is non-constant, then it is also a bipolar fuzzy prime ideal of $X$.

Proof. Let $\Psi=\left(X ; \mu_{\Psi}^{P}, \mu_{\Psi}^{N}\right)$ be a bipolar fuzzy prime ideal of an implicative BCK-algebra $X$ such that $\langle\Psi,(a, b)\rangle=\left(X ;\langle\mu, a\rangle_{\Psi}^{P},\langle\mu, b\rangle_{\Psi}^{N}\right)$ is non-constant. Clearly, $\langle\Psi,(a, b)\rangle$ is a bipolar fuzzy set of $X$. Let $x, y \in X$. Since $\Psi=\left(X ; \mu_{\Psi}^{P}, \mu_{\Psi}^{N}\right)$ is a bipolar fuzzy prime ideal $X$, we have

$$
\begin{aligned}
\langle\mu, a\rangle_{\Psi}^{P}(x \wedge y) & =\mu_{\Psi}^{P}((x \wedge y) * a) \\
& =\mu_{\Psi}^{P}((x * a) \wedge(y * a)) \\
& \leq \max \left\{\mu_{\Psi}^{P}(x * a), \mu_{\Psi}^{P}(y * a)\right\} \\
& =\max \left\{\langle\mu, a\rangle_{\Psi}^{P}(x),\langle\mu, a\rangle_{\Psi}^{P}(y)\right\}
\end{aligned}
$$

and

$$
\begin{aligned}
\langle\mu, b\rangle_{\Psi}^{N}(x \wedge y) & =\mu_{\Psi}^{N}((x \wedge y) * b) \\
& =\mu_{\Psi}^{N}((x * b) \wedge(y * b)) \\
& \geq \min \left\{\mu_{\Psi}^{N}(x * b), \mu_{\Psi}^{N}(y * b)\right\} \\
& =\min \left\{\langle\mu, b\rangle_{\Psi}^{N}(x),\langle\mu, b\rangle_{\Psi}^{N}(y)\right\} .
\end{aligned}
$$

Thus, the extension $\langle\Psi,(a, b)\rangle=\left(X ;\langle\mu, a\rangle_{\Psi}^{P},\langle\mu, b\rangle_{\Psi}^{N}\right)$ of $\Psi$ by $(a, b)$ is a bipolar fuzzy prime ideal of $X$. This completes the proof.

\section{Bipolar fuzzy commutative ideals}

Definition 4.1. A bipolar fuzzy set $\Psi=\left(X ; \mu_{\Psi}^{P}, \mu_{\Psi}^{N}\right)$ in $X$ is called a bipolar fuzzy commutative ideal of $X$ if it satisfies (BI1) and

(BC1) $\mu_{\Psi}^{P}(x *(y *(y * x))) \geq \min \left\{\mu_{\Psi}^{P}((x * y) * z), \mu_{\Psi}^{P}(z)\right\}$,

(BC2) $\mu_{\Psi}^{N}(x *(y *(y * x))) \leq \max \left\{\mu_{\Psi}^{N}((x * y) * z), \mu_{\Psi}^{N}(z)\right\}$, for all $x, y, z \in X$.

Proposition 4.2. Every bipolar fuzzy commutative ideal of $X$ is a bipolar fuzzy ideal of $X$. 
Proof. Let $\Psi=\left(X ; \mu_{\Psi}^{P}, \mu_{\Psi}^{N}\right)$ be a bipolar fuzzy commutative ideal of $X$. Clearly, $\Psi$ satisfies the condition (BI1). Let $y=0$ in both (BC1) and (BC2). Then $\mu_{\Psi}^{P}(x) \geq \min \left\{\mu_{\Psi}^{P}(x * z), \mu_{\Psi}^{P}(z)\right\}$ and $\mu_{\Psi}^{N}(x) \leq$ $\max \left\{\mu_{\Psi}^{N}(x * z), \mu_{\Psi}^{N}(z)\right\}$, for all $x, z \in X$. Hence, $\Psi$ satisfies conditions (BI2) and (BI2). Thus, $\Psi=\left(X ; \mu_{\Psi}^{P}, \mu_{\Psi}^{N}\right)$ is a bipolar fuzzy ideal of $X$. This completes the proof.

A bipolar fuzzy ideal of a BCK-algebra $X$ may not be a bipolar fuzzy commutative ideal of $X$.

EXAmple 4.3. Let $X=\{0,1,2,3,4\}$ in which $*$ is defined by:

\begin{tabular}{c|ccccc}
$*$ & 0 & 1 & 2 & 3 & 4 \\
\hline 0 & 0 & 0 & 0 & 0 & 0 \\
1 & 1 & 0 & 1 & 0 & 0 \\
2 & 2 & 2 & 0 & 0 & 0 \\
3 & 3 & 3 & 3 & 0 & 0 \\
4 & 4 & 4 & 4 & 3 & 0
\end{tabular}

Then $(X ; *, 0)$ is a BCK-algebra. Define mappings $\mu_{\Psi}^{P}: X \rightarrow[0,1]$ and $\mu_{\Psi}^{N}: X \rightarrow[-1,0]$ by

$$
\mu_{\Psi}^{P}=\left(\begin{array}{ccccc}
0 & 1 & 2 & 3 & 4 \\
1 & 0.7 & 0.3 & 0.3 & 0.3
\end{array}\right)
$$

and

$$
\mu_{\Psi}^{N}=\left(\begin{array}{ccccc}
0 & 1 & 2 & 3 & 4 \\
-0.9 & -0.5 & -0.1 & -0.1 & -0.1
\end{array}\right) .
$$

Then $\Psi=\left(X ; \mu_{\Psi}^{P}, \mu_{\Psi}^{N}\right)$ is a bipolar fuzzy ideal of $X$. But it is not a bipolar fuzzy commutative ideal of $X$, because $\mu_{\Psi}^{P}(2 *(3 *(3 * 2))) \not$ $\min \left\{\mu_{\Psi}^{P}((2 * 3) * 0), \mu_{\Psi}^{P}(0)\right\}$.

Theorem 4.4. Let $\Psi=\left(X ; \mu_{\Psi}^{P}, \mu_{\Psi}^{N}\right)$ be a bipolar fuzzy ideal of $X$. Then it is a bipolar fuzzy commutative ideal of $X$ if and only if it satisfies the conditions

(a) $\mu_{\Psi}^{P}(x *(y *(y * x))) \geq \mu_{\Psi}^{P}(x * y)$,

(b) $\mu_{\Psi}^{N}(x *(y *(y * x))) \leq \mu_{\Psi}^{N}(x * y)$,

for all $x, y \in X$.

Proof. $(\Rightarrow)$ It is straightforward by taking $z=0$ in Definition 4.1.

$(\Leftarrow)$ Let $\Psi=\left(X ; \mu_{\Psi}^{P}, \mu_{\Psi}^{N}\right)$ be a bipolar fuzzy ideal of $X$. Assume that it satisfies the condisions $(a)$ and $(b)$. Let $x, y, z \in X$. Then

$$
\begin{aligned}
\mu_{\Psi}^{P}(x *(y *(y * x))) & \geq \mu_{\Psi}^{P}(x * y) \\
& \geq \min \left\{\mu_{\Psi}^{P}((x * y) * z), \mu_{\Psi}^{P}(z)\right\} .
\end{aligned}
$$


Similarly, we have $\mu_{\Psi}^{N}(x *(y *(y * x))) \leq \max \left\{\mu_{\Psi}^{N}((x * y) * z), \mu_{\Psi}^{N}(z)\right\}$, for all $x, y, z \in X$. Thus, $\Psi=\left(X ; \mu_{\Psi}^{P}, \mu_{\Psi}^{N}\right)$ is a bipolar fuzzy commutative ideal of $X$. This completes the proof.

Theorem 4.5. Every bipolar fuzzy ideal of a commutative BCKalgebra is a bipolar fuzzy commutative ideal of $X$.

Proof. Let $\Psi=\left(X ; \mu_{\Psi}^{P}, \mu_{\Psi}^{N}\right)$ be a bipolar fuzzy ideal of a commutative BCK-algebra $X$. Let $x, y, z \in X$. Then

$$
\begin{aligned}
\mu_{\Psi}^{P}(x *(y *(y * x))) & =\mu_{\Psi}^{P}(x *(x *(x * y))) \\
& \geq \mu_{\Psi}^{P}(x * y) \\
& \geq \min \left\{\mu_{\Psi}^{P}((x * y) * z), \mu_{\Psi}^{P}(z)\right\} .
\end{aligned}
$$

Similarly, we have $\mu_{\Psi}^{N}(x *(y *(y * x))) \leq \max \left\{\mu_{\Psi}^{N}((x * y) * z), \mu_{\Psi}^{N}(z)\right\}$, for al $x, y, z \in X$. Thus, $\Psi=\left(X ; \mu_{\Psi}^{P}, \mu_{\Psi}^{N}\right)$ is a bipolar fuzzy commutative ideal of $X$. This completes the proof.

Theorem 4.6. Let $\Psi=\left(X ; \mu_{\Psi}^{P}, \mu_{\Psi}^{N}\right)$ be a bipolar fuzzy commutative ideal of a positive implicative $B C K$-algebra $X$. Then the extension $\langle\Psi,(a, b)\rangle=\left(X ;\langle\mu, a\rangle_{\Psi}^{P},\langle\mu, b\rangle_{\Psi}^{N}\right)$ of by $(a, b)$ is a bipolar fuzzy commutative ideal of $X$.

Proof. Let $\Psi=\left(X ; \mu_{\Psi}^{P}, \mu_{\Psi}^{N}\right)$ be a bipolar fuzzy commutative ideal of a positive implicative BCK-algebra $X$ and let $a, b \in X$.

$$
\begin{aligned}
\langle\mu, a\rangle_{\Psi}^{P}(x *(y *(y * x))) & =\mu_{\Psi}^{P}((x *(y *(y * x))) * a) \\
& =\mu_{\Psi}^{P}(((x * a) *((y * a) *((y * a) *(x * a))))) \\
& \geq \mu_{\Psi}^{P}((x * a) *(y * a)) \\
& =\mu_{\Psi}^{P}((x * y) * a) \\
& =\langle\mu, a\rangle_{\Psi}^{P}(x * y)
\end{aligned}
$$

and

$$
\begin{aligned}
\langle\mu, a\rangle_{\Psi}^{N}(x *(y *(y * x))) & =\mu_{\Psi}^{N}((x *(y *(y * x))) * a) \\
& =\mu_{\Psi}^{N}(((x * a) *((y * a) *((y * a) *(x * a))))) \\
& \leq \mu_{\Psi}^{N}((x * a) *(y * a)) \\
& =\mu_{\Psi}^{N}((x * y) * a) \\
& =\langle\mu, a\rangle_{\Psi}^{N}(x * y),
\end{aligned}
$$


for all $x, y \in X$. Thus, it follows from Theorem 4.4 that the exten$\operatorname{sion}\langle\Psi,(a, b)\rangle=\left(X ;\langle\mu, a\rangle_{\Psi}^{P},\langle\mu, b\rangle_{\Psi}^{N}\right)$ of $\Psi$ by $(a, b)$ is a bipolar fuzzy commutative ideal of $X$. This completes the proof.

\section{References}

[1] Y. Imai and K. Iséki, On axiom systems of propositional calculi XIV, Proc. Japan Academy 42 (1966), 19-22.

[2] K. Iséki and S. Tanaka, An introduction to theory of BCK-algebras, Math. Japon. 23 (1978), 1-26.

[3] W. K. Jeong, Fuzzy ideal extensions of BCK-algebras, Far East J. Math. Sci. 7(1) (2002), 67-75.

[4] Y. B. Jun, Fuzzy topological BCK-algebras, Math. Japon. 38 (1993), 1059-1063.

[5] K. J. Lee, Bipolar fuzzy subalgebras and bipolar fuzzy ideals of BCK/BCIalgebras, Bull. Malays. Math. Sci. Soc. 32 (2009), 361-373.

[6] K. M. Lee, Bipolar-valued fuzzy sets and their operations, Proc. Int. Conf. on Intelligent Technologies, Bangkok, Thailand (2000), 307-312.

[7] J. Meng and Y. B. Jun, BCK-algebras, Kyungmoonsa, Seoul, Korea, 1994.

[8] O. G. Xi, Fuzzy BCK-algebras, Math. Japon. 36 (1991), 935-942.

[9] X. Y. Xie, Fuzzy ideals extensions of semigroups, Soochow J. Math. 27 (2001), 125-138.

[10] L. A. Zadeh, Fuzzy sets, Inform and Control. 8 (1965), 338-353.

*

Faculty of Liberal Education

Kyungpook National University

Daegu 702-701, Republic of Korea

E-mail: wkjeong@knu.ac.kr 